\title{
Mechanisms of high-intensity sound exposure on inhibiting hippocampal long-term potentiation: role of brain-derived neurotrophic factor.
}

Júnia L. de Deus ${ }^{1}$, Mateus R. Amorim², Aline B. Ribeiro ${ }^{1}$, Procópio C. G. Barcellos-Filho ${ }^{1}$, César C. Ceballos ${ }^{2}$, Luiz Guilherme S. Branco ${ }^{2}$, Alexandra O.S. Cunha ${ }^{1}$, Ricardo M. Leão ${ }^{1^{*}}$.

${ }^{1}$ Department of Physiology, School of Medicine of Ribeirão Preto, University of São Paulo, Ribeirão Preto-SP, 14049-900, Brazil.

2 Department of Basic and Oral Biology, Dental School of Ribeirão Preto, University of São Paulo, Ribeirão Preto-SP, 14040-904, Brazil.

*Correspondence to: Ricardo M. Leão, leaor@fmrp.usp.br. ORCID: 0000-0002-3829-3342

Conflict of Interests: The authors declare no competing interests. 


\begin{abstract}
:
Exposure to humans and experimental animals to loud noises produce cognitive and emotional disorders and recent studies have shown that hippocampal neuronal function is affected by auditory stimulation or deprivation. We have found previously that in the hippocampus of rats exposed to high-intensity sound $(110 \mathrm{~dB})$ for one-minute the Schaffer-CA1 long-term potentiation (LTP) is strongly inhibited. Here we investigated possible mechanisms involved in this effect. We found, using c-fos expression, that exposure to $110 \mathrm{~dB}$ sound-activated neurons in the CA1 and CA3 hippocampal region. Using electrophysiological recordings in hippocampal slices, we found that both GABAergic and glutamatergic neurotransmission were unaffected by high-intensity sound stimulation. However, hippocampal brain-derived neurotrophic factor (BDNF), which is involved in promoting hippocampal synaptic plasticity, presented decreased levels in sound-stimulated animals. Perfusion of slices with BDNF revert the inhibition of LTP after a single sound stimulus in comparison to sham-stimulated rats. Furthermore, the perfusion with LM 22A4, a TrkB receptor agonist also rescued LTP from sound-stimulated animals. Our results strongly suggest that the exposure to high-intensity sound inhibits the BDNF production in the hippocampus, which could be a possible mechanism of the inhibition of LTP by highintensity sound exposure.
\end{abstract}

Keywords: hippocampus, BDNF, LTP, high-intensity sound. 


\section{Introduction}

Loud sounds can damage cochlear hair cells and is currently the leading cause of permanent hearing loss and tinnitus (Le et al., 2017). Even prolonged exposure to non-traumatic noise has adverse effects on the auditory processing (Eggermont et al., 2017) and exposure to moderate to high-intensity sound triggers behavior responses resulting in the avoidance of the sound source (Manohar et al., 2017), a protective measure to avoid damage to auditory hair cells. Indeed, loud sounds are stressors that lead to the activation of the HPA axis, leading to increased secretion of corticosterone (Helfferich and Palkovits, 2003; Burrow et al., 2005). Thus, non-auditory areas are affected by the level of auditory input and adjust the animal's behavior to the acoustic environment.

Additionally, exposure to loud and even moderate sounds can produce several systemic and cognitive deficits in humans (Lercher et al., 2003; Stansfeld et al., 2005; Basner et al., 2014). The so-called "sonic attack" to the American and Canadian diplomatic personnel in Havana, Cuba, which produced several emotional and cognitive symptoms after the exposure to a highpitched loud sound (Hofner et al., 2018; Swanson et al., 2018), is a dramatic example of the potential harm that loud sound exposure can accomplish beyond the auditory system.

The hippocampus receives sensory information from several modalities, which are fundamental for its role in spatial navigation, learning and memory formation (Save et al., 2000; Jeffery, 2007; Ravassard et al., 2013). It is connected to the auditory system (Kraus and Canlon, 2012; Zhang et al., 2018) and sound stimulation triggers excitatory and inhibitory neurotransmission in hippocampal neurons in vivo (Wang et al., 2017). Also, the hippocampus is related to the formation of auditory memories (Squire et al., 2001), it uses auditory information for the formation of spatial memory (Tamura et al., 1990) and hippocampal place cells can be activated by auditory dimension cues (Aronov et al., 2017). On the other hand, prolonged exposure to loud or even moderate-intensity sounds can affect the hippocampus negatively. In this case, prolonged exposure to moderate-intensity sound increases oxidative damage and tau phosphorylation in the hippocampus and impairs spatial memory in mice (Cheng et al., 2011,2016), whereas high-intensity sound exposure affects hippocampal place cells activity (Goble et al., 2009). Additionally, exposure to moderate to loud sounds can lead to impairment of spatial and associative memory, which seems to be associated to an oxidative status imbalance (Uran et al., 2010, 2012; Manikandan et al., 2016). On the other hand, 40minutes exposure to mild ( $80 \mathrm{~dB}$ ) sounds, potentiated hippocampal long-term potentiation (LTP) and brain-derived neurotrophic factor (BDNF) expression (Matt et al., 2018). Finally, we have shown that one minute exposure to a high-intensity (110-120 dB) broadband sound inhibits LTP in the Schaffer-CA1 synapses in the hippocampus of rats for 24 hours after exposure (de Deus et al., 2017), an effect not related to corticosterone secretion, and not observed after moderate noise $(80 \mathrm{~dB}$ ) exposure.

Hippocampal LTP is a long-lasting enhancement of synaptic efficacy associated with hippocampal learning and memory (Bliss and Lomo, 1973; Bliss and Collingridge, 1993). In the Schaffer-CA1 synapse, the LTP is dependent on post-synaptic calcium influx via glutamatergic NMDA receptors (for review see Malenka and Bear, 2004; Nicoll, 2017). LTP is modulated positively or negatively by several signaling molecules, such as BDNF, a neurotrophic factor which acts on TrkB receptors (Minichielo, 2009). Activation of TrkB receptors by BDNF promotes LTP in the Schaffer-CA1 synapse (Minichielo, 2009; Edelman et al., 2014; Lin et al., 2018). Heterozygous BDNF (+/-) knockout mice have a significant deficit in hippocampal LTP (Korte et al., 1995), which can be rescued by exogenous BDNF (Paterson et al., 1996). The secretion of BNDF is also promoted by stimulation protocols that develop LTP in contrast to stimulation patterns that do not lead to LTP (Aicardi et al., 2004). In accordance to the role of BDNF in facilitating the induction of LTP, BDNF can revert LTP in situations where the LTP is inhibited by 
exogenous conditions, like after prolonged periods of chronic intermittent hypoxia (Xie et al., 2010).

Here we investigated the mechanisms of LTP inhibition by a single episode of highintensity sound stimulation (de Deus et al., 2017). We demonstrated that both glutamatergic and GABAergic transmission is unaffected by a single episode of high intense sound stimulation, but the levels of BDNF in the hippocampus are reduced, and LTP could be rescued by exogenous BDNF application.

\section{Materials and Methods:}

All experimental procedures involving animals were elaborated according to the rules of research in the National Council for Control of Animal Experimentation and approved by the Committee on Ethics in Animal Use of the Ribeirão Preto Medical School of the University of São Paulo, (protocol \# 006/2-2015).

\section{Animals}

Experiments were performed on male Wistar rats 60-70 days old obtained from the Central Animal Facility of the Ribeirão Preto Campus of the University of São Paulo. Rats were kept in Plexiglas cages (2-3 animals per cage) at the Medical School of Ribeirão Preto, with food and water available ad libitum and a 12-hr dark/light cycle (lights on at 7:00 a.m.) and controlled temperature $\left(22^{\circ} \mathrm{C}\right)$. We divided the animals in two experimental groups: sham-stimulated (rats placed in the sound stimulus box without sound stimulus) and rats submitted to a sound stimulus of $110 \mathrm{~dB}$ (high-intensity sound).

\section{Sound stimulation}

Rats were placed in an acrylic arena (height: $32 \mathrm{~cm}$, diameter: $30 \mathrm{~cm}$ ), located inside an acoustically isolated chamber $(45 \times 45 \times 40 \mathrm{~cm})$, with 2 loudspeakers placed at the top of the arena, in accordance with protocols described previously by de Deus et al., (2017). After oneminute acclimatization, they were submitted to a one-minute duration stimulus of $110 \mathrm{~dB}$, consisting of a digitally modified recording of a doorbell, spanning frequencies from 3 to $15 \mathrm{kHz}$ (Romcy-Pereira and Garcia-Cairasco, 2003). After stimulation, animals were kept in the arena for one more minute and returned to their boxes where they remained for two hours until the preparation of the hippocampal slices. Sham-stimulated animals were placed in the same arena for 3 minutes without any sound stimulus. Ambient noise inside the acoustic chamber was 55 $\mathrm{dB}$, and the sound intensity inside the arena was checked and calibrated regularly with a decibel meter (Extech 407730 - Sound Level Meter).

\section{c-Fos Immunofluorescence}

After 90 minutes of the sound or sham stimulus, rats were anesthetized with isoflurane for transcardial perfusion with phosphate buffered saline (PBS, $0.1 \mathrm{M}, \mathrm{pH} 7.4$ ) and paraformaldehyde at $4 \%$ dissolved in PBS. After perfusion, the brains were immediately removed and immersed in 30\% sucrose solution in PBS until tissue saturation. Next, tissue blocks were frozen in isopentane for $30 \mathrm{~s}$ and stored at $-80^{\circ} \mathrm{C}$ until sectioned. Coronal sections of 40 $\mu \mathrm{m}$ were cut, in a cryostat, in four series in the CA1 region. The sections were washed rapidly with glycine $(0.1 \mathrm{M})$ to remove excess paraformaldehyde, permeabilized with $0.1 \%$ Triton X-100 for 10 minutes and washed with 0.1M PBS. Nonspecific binding was blocked with PBS containing $1 \%$ bovine serum albumin (BSA; Sigma, St. Louis, MO, USA) for 1 hour. Subsequently, sections were incubated for 40 hours at $4^{\circ} \mathrm{C}$ with a polyclonal primary anti-c-Fos antibody produced in rabbit (1: 5000; Santa Cruz Biotechnology, Dallas, TX, USA). The primary antibody was diluted in PBS containing $0.1 \%$ Triton X-100 and 1\% BSA. Then, after washing with PBS (5X for $5 \mathrm{~min}$ ), 
sections were incubated with biotinylated anti-goat antibody IgG produced in rabbit (1: 1000; Alexa 594, Vector Laboratories, Burlingame, CA, USA) for 1 hour. Finally, nuclear labeling was done with DAPI (4', 6-diamidino-2-phenylindole; $1 \mu \mathrm{g} / \mathrm{mL}$, Sigma-Aldrich) for 10 minutes at room temperature. Sections were then mounted on slides using Fluoromount $G$ (Electron Microscopy Sciences, Hatfield, PA) as assembling medium, viewed and photographed under fluorescence microscopy DM 5500B (Leica Microsystems, Wetzlar, Germany). Negative control of the primary antibody was performed, where they were omitted, and the cuts were only incubated with the secondary antibodies. No labeling was observed on these controls.

\section{Preparation of Hippocampal Slices}

After two hours of the sound or sham stimulus, the animals were anesthetized with isoflurane and decapitated. The brains were rapidly removed and placed in an ice-cold solution containing: $87 \mathrm{NaCl}, 2.5 \mathrm{KCl}, 25 \mathrm{NaHCO}_{3}, 1.25 \mathrm{NaH}_{2} \mathrm{PO}_{4}, 75$ sucrose, 25 Glucose, $0.2 \mathrm{CaCl}_{2}, 7$ $\mathrm{MgCl}_{2}$, bubbled with $95 \% \mathrm{O}_{2}$ and $5 \% \mathrm{CO}_{2}$. Brain hemispheres were separated, positioned side by side, fixed with cyanoacrylate glue (SuperBonder ${ }^{\circledR}$ ) to a base and placed in the vibrating chamber of a vibratome (1000 plus, Vibratome, USA). Transversal slices containing the dorsal hippocampus (200 $\mu \mathrm{m}$ for whole-cell recordings and $400 \mu \mathrm{m}$ for extracellular recordings) cut and the hippocampus was separated from the cortex using ophthalmic scissors and microtweezers. Slices were placed in artificial cerebrospinal fluid (aCSF) solution containing (mM): 125 $\mathrm{NaCl}, 2.5 \mathrm{KCl}, 1.25 \mathrm{NaH}_{2} \mathrm{PO}_{4}, 26 \mathrm{NaHCO}_{3}, 10 \mathrm{Glucose}, 2 \mathrm{CaCl}_{2}, 1 \mathrm{MgCl}_{2}$ and left to rest for at least two hours before use (one hour at $34-35{ }^{\circ} \mathrm{C}$ and at least one hour in room temperature) continuously bubbled with a carbogenic mixture $\left(95 \% \mathrm{O}_{2}\right.$ and $\left.5 \% \mathrm{CO}_{2}\right)$.

\section{Whole-Cell Patch-Clamp Recordings}

Whole-cell patch-clamp experiments were performed in slices placed in a chamber continuously perfused aCSF $(1 \mathrm{ml} / \mathrm{min})$ supplemented with picrotoxin $\left(G A B A_{A}\right.$ receptor antagonist: $20 \mu \mathrm{M}$ ) except for GABAergic neurotransmission, and at a temperature of $34{ }^{\circ} \mathrm{C}$ controlled with inline heater (Scientifica). Pyramidal neurons from the CA1 region were visualized with an Olympus BX51WI microscope through a 40x water immersion objective and with infrared differential interference contrast (IR-DIC). Pyramidal cells were chosen according to the morphology (pyramidal shape) and position, in the middle of the pyramidal layer. Recording electrodes were filled with an internal pipette solution chosen for each set of experiments and fabricated from borosilicate capillaries (BF150-86-10, 15 Sutter Instruments) with the tip resistance between 4-5 $\mathrm{M} \Omega$. Electrophysiological recordings in whole-cell patchclamp were performed using a Heka EPC10 amplifier, with a sampling rate of $50 \mathrm{kHz}$ and low pass filtered at $3 \mathrm{kHz}$ (Bessel). Neurons that presented series resistance increased over $20 \%$ during experiments, as well as resting membrane potential higher than $-60 \mathrm{mV}$, were discarded. Series resistance $(<20 \mathrm{M} \Omega)$ was compensated at around $60 \%$. Voltages were corrected off-line for a liquid junction potential for each internal solution calculated with Clampex software (Molecular Devices).

Glutamatergic EPSCS were evoked from the stimulation of the Schaffer pathway with a concentric bipolar microelectrode (FHC-Bowdoin, ME, USA) connected to an SD9 Grass voltage stimulator (Natus Medical Incorporated, Warwick, RI, USA). AMPA and NMDA-EPSCs recording were performed under the perfusion of the slices with picrotoxin $(20 \mu \mathrm{M})$ using an internal solution composed in mM: $130 \mathrm{CsCl}, 10 \mathrm{HEPES}, 5 \mathrm{EGTA}, 5$ phosphocreatine, $4 \mathrm{Mg}$-ATP, $0.5 \mathrm{Na}$ GTP, 10 TEA, 5 QX 314, adjusted to $\mathrm{pH} 7.3$ with $\mathrm{CsOH}$ and $\approx 290 \mathrm{mOsm} / \mathrm{kgH}_{2} \mathrm{O}$. AMPA and NMDA currents were recorded at potentials from -70 to $+80 \mathrm{mV}$ with increments of $+30 \mathrm{mV}$. The stimulus intensity was gradually increased until the amplitude of the synaptic current reached its maximum. We then stimulated Schaffer pathway with the minimum voltage necessary to evoke a maximum post-synaptic current for recording the currents. To obtain NMDA currents, 
we blocked AMPA/KA currents with DNQX $(10 \mu \mathrm{M})$ for 10 minutes and to estimate AMPA currents, we subtracted currents before and after DNQX.

Miniature GABAergic IPSCs (mIPSCs) were recorded for 10 minutes at $-70 \mathrm{mV}$ with an internal solution consisting in (mM): $145 \mathrm{KCl}, 10 \mathrm{HEPES}, 0.5 \mathrm{EGTA}, 10$ phosphocreatine, $4 \mathrm{Mg}$ ATP, $0.3 \mathrm{Na}-\mathrm{GTP}$, adjusted to $\mathrm{pH} 7.3$ with $\mathrm{KOH}$ and $\approx 290 \mathrm{mOsm} / \mathrm{kg} \mathrm{H}_{2} \mathrm{O}$. Miniature IPSCs (mIPSC) were recorded in aCSF in the presence of TTX $(0.5 \mu \mathrm{M})$ and DNQX $(10 \mu \mathrm{M})$.

\section{BDNF quantification}

BNDF was quantified using ELISA. In these experiments, 4 hours after sham or sound stimulus, rats were decapitated and the brains were carefully collected, frozen by submersion in dry ice-cold isopentane and kept under $-80^{\circ} \mathrm{C}$. The dorsal hippocampus was sampled in a cryostat by a punch needle ( $1.5 \mathrm{~mm}$ inner diameter) from a $1200-\mu \mathrm{m}$ thick slice (Figure $4 \mathrm{~A})$ and stored in plastic tubes at $-80{ }^{\circ} \mathrm{C}$ until analysis. Samples were homogenized with buffer solution (137 mM NaCl, 20 mM Tris- $\mathrm{HCl}$ pH 7.6, 10\% glycerol and sodium orthovanadate), supplemented with phosphatase protease inhibitor cocktail (Cell Signaling, Massachusetts, USA) and centrifuged at $13.000 \mathrm{rpm}$ for $20 \mathrm{~min}$ at $4{ }^{\circ} \mathrm{C}$. Tissue supernatant were used to estimate BDNF (detection limits 7.8-500 pg/ml) by ELISA (BDNF Emax Immuno Assay System, \#G7611, Promega), according to the manufacturer's instructions. Results from BDNF in hippocampal homogenates were normalized by protein concentrations, which were assessed by the Bradford assay (\#5000205, Bio-Rad Laboratories, USA).

\section{Field potential recordings and LTP induction}

Extracellular electrophysiological recordings were performed with a Multiclamp 700B amplifier (Molecular Devices, USA) connected to a Digidata 1440 A AC/DC interface (Molecular Devices, USA). Slices were placed in the recording chamber with continuous superfusion of aCSF $(1 \mathrm{~mL} / \mathrm{min})$ bubbled with a carbogenic mixture and the temperature-controlled $\left(32-34^{\circ} \mathrm{C}\right)$ using an inline temperature heater (Warner Instruments, USA). To stimulate Schaffer/collateral fibers, we used a stainless steel bipolar concentric microelectrode (FHC-Bowdoin, Maine, USA) connected to a Master-9 voltage stimulator (A.M.P.I., Israel). Field excitatory post-synaptic potentials (fEPSPs) were recorded at CA1 stratum radiatum with borosilicate glass microelectrodes (G85150T, Warner Instruments, USA) filled with aCSF with tip resistances of 1$2 \mathrm{M} \Omega$. For LTP induction, first, we performed an input-output curve, where the voltage was gradually increased by $10 \mathrm{~V}$ increments until population spikes were observed in the fEPSP. From the maximum stimulus, we choose the stimulus intensity that produced a fEPSP equivalent to approximately $50 \%$ of the maximum response. Subsequently, 50 fEPSPs were recorded at this intensity, for 25 minutes at $0.03 \mathrm{~Hz}$. After baseline recording, LTP was induced on Schaffercollateral fibers using 3 trains of high-frequency stimulation (HFS) at $100 \mathrm{~Hz}, 1$-second duration (inter-train interval of $3 \mathrm{~s}$ ) and after that, we recorded fEPSPs for 80 minutes. BDNF ( $25 \mathrm{ng} / \mathrm{ml}$ ) and (LM-22A4: $5 \mu \mathrm{M}$ ) were perfused in the last 5 minutes of the baseline and in the first 5 minutes post-LTP. Signals were acquired at $100 \mathrm{kHz}$, filtered at $3 \mathrm{kHz}$ (Bessel, 8-pole) with pClamp 10.2 software (Molecular Devices, USA).

\section{Data Analysis and Statistics}

For quantitative analysis of cells positive for c-fos, two consecutive sections containing the hippocampus were taken from each animal, blindly. In each section, the number of c-Fos-positive cells was counted bilaterally using an automated cell counting procedure by ImageJ software (National Institute of Health, Bethesda, MA). The image was converted to grayscale using the thresholding feature, to highlight and standard the shape parameters of the immunoreactive particles so that the program determined what to consider a cell. Threshold settings and light intensity were kept constant across all sections and during photo acquisition. 
The thresholded Fos-stained cells for the area were averaged and are presented as Fos-positive nuclei/section for each brain area.

AMPA and NMDA EPSCs slope conductances were determined as the slope of linear functions of the most linear parts of the IV relationships. mIPSCs were analyzed using Mini Analysis software (Synaptosoft 6.0.3, Fort Lee, NJ, USA) and EPSCs with custom-written routines in IgorPro (Wavemetrics, Portland, OR, USA) and Matlab (MathWorks, Natick, MA, USA). The peaks of the EPSCS were used to build IV relationships to calculate the reversal potential. We measured rise times from baseline to peak and decay times from peak to baseline. mIPSCs were recorded for 10 minutes at $-70 \mathrm{mV}$ after the application of $T T X$ to block action potentials and were selected manually, and only currents with good signal to noise ratio which could be easily identified as mIPSCs were chosen. Histograms for inter-event (IEI) intervals, amplitudes and rise times were built with the same fixed bins for different groups of cells and. Analysis of decay kinetics for inhibitory currents was performed by Mini Analysis group analysis with individual currents fitted with double exponential functions. Fast and slow time constants were presented as the average and compared between groups:

Field potentials were analyzed with Clampfit 10.2. Traces were low-pass filtered offline $(500 \mathrm{~Hz})$, and the slopes of the fEPSPs were fitted with a standard linear function. Data from each experiment were normalized relative to its baseline. LTP was quantified as the average of the fEPSP slopes in the last 20 minutes from 80 minutes post-induction recording period.

All the results are presented as means $\pm \mathrm{SE}$, and statistical significance was determined using unpaired Student's t-test and one-way ANOVA as required, cumulative frequency distributions were tested for significance with Kolmogorov-Smirnov (KS) test. We use a significance level of $5 \%(P \leq 0.05)$.

\section{Results}

High-intensity sound exposure increases c-fos expression in hippocampal neurons.

Because high-intensity sound affects the hippocampus in several ways (de Deus at al., 2017; Cunha et al., 2019), we tested if our protocol of high-intensity sound stimulation was able to activate hippocampal cells through of c-FOS expression. C-FOS is an immediate early gene (IEG) which expression is driven by calcium entry by NMDA receptor activation and voltagedependent calcium channels, during intense action potential firing, and it is used as a marker of neuronal activation (Dragunow and Faull, 1989; Hudson et al., 2018). Immunostaining for c-FOS in stimulated rats showed a high level of c-FOS expression in CA1 neurons in the pyramidal layer (192.0 \pm 16.71 cells/section; $n=3$ animals) compared to sham-stimulated (142.0 \pm 3.041 cells/section; $n=3$ animals; $P=0.04$, Figure $1 \mathrm{~A}$ ). We also observed that pyramidal layer CA3 neurons presented higher c-FOS expression in stimulated $(154.8 \pm 12.74$ cells/section; $n=3$ animals) when compared sham-stimulated rats ( $88.50 \pm 5.074$ cells/section; $n=3$ animals; $P=$ 0.008 , Figure 1B). In contrast, c-FOS expression was similar in neurons of the dentate gyrus, in sham and stimulated rats $(P \geq 0.05$, Figure $1 C)$. These findings indicate that high-intensity sound drives robust excitatory synaptic activity, which is able to induce c-FOS expression in hippocampal neurons. 

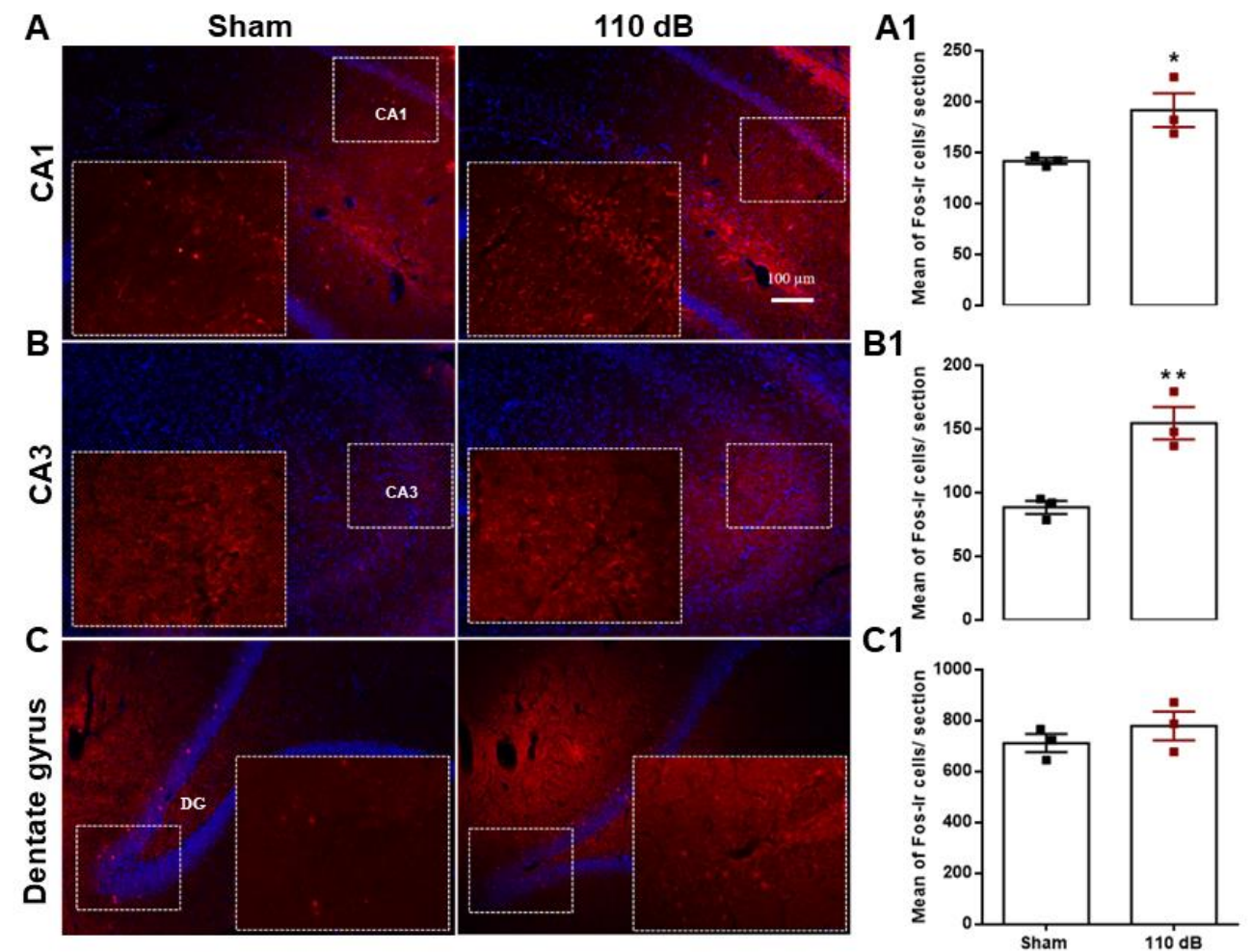

Figure 1. Photomicrographs illustrating c-FOS-positive nuclei labeling (red) and cell nuclei stained with DAPI (blue) in sections of CA1 (A), CA3 (B) and dentate gyrus (C). Left panel, representative images from an animal exposed to sham stimulation and an animal exposed to $110 \mathrm{~dB}$ sound stimulation (10X). Inset: squared area at higher magnification (20X). Right panel, the number of c-FOS-positive nuclei staining/section for CA1 (A1), CA3 (B1) and dentate gyrus (C1). ${ }^{*} \mathrm{P}<0.05$.

High-intensity sound exposure does not affect glutamatergic neurotransmission in the SchafferCA1 synapses.

LTP is dependent on the activation of NMDA receptors in the synapses of the SchafferCA1 pathway with pyramidal neurons of the hippocampus, (Collingridge and Bliss, 1987; Tang et al., 1999 and Tsien et al., 1996). The high-intensity sound induced inhibition of LTP previously shown by our group (de Deus, et al, 2017) could be due to an inhibition of NMDA-mediated currents or to a decreased AMPA/Kainate (AMPA/KA) receptor activation. Therefore, we investigated if acute high-intensity sound stimulus alters glutamatergic synaptic neurotransmission. In these experiments, we recorded AMPA/KA (DNQX-sensitive) and NMDA (DNQX-resistant) receptor-mediated EPSCs from Schaffer-CA1 synapses from sham and stimulated animals (Figure 2A). Both IV relationships of the AMPA/KA and NMDA receptor mediated EPSCs were similar between the sham and stimulated groups (Figure 2B and 2C). The calculated slope conductance of the currents was not significant different between sham and stimulated groups (AMPA/KA, sham: $8.4 \pm 0.6 \mathrm{nS}$; stimulated: $8.4 \pm 0.7 \mathrm{nS}, \mathrm{p}=0.9$; NMDA, sham: $3.8 \pm 0.3 \mathrm{nS}$; stimulated: $4.0 \pm 0.4 \mathrm{nS}, \mathrm{p}=0.6)$. The amplitudes of AMPA/KA receptor-mediated EPSCs at $-80 \mathrm{mV}$ in sham $(-616.4 \pm 101.7 \mathrm{pA} ; \mathrm{n}=9)$ and stimulated animals $(-657.2 \pm 108.2 \mathrm{pA} ; \mathrm{n}$ $=16$ ) were not see significantly different $(p=0.80$;Figure 2D). Also NMDA-mediated EPSCs at $+70 \mathrm{mV}$, were not significantly different between sham $(310.2 \pm 56.59 \mathrm{pA} ; \mathrm{n}=9)$ and stimulated 
groups ( $370.7 \pm 71.43 \mathrm{pA} ; \mathrm{n}=11 ; \mathrm{p}=0.52$; Figure $2 \mathrm{E}$ ). Our data show that a sound stimulus of $110 \mathrm{~dB}$ of 1-minute duration does not alter fast hippocampal glutamatergic neurotransmission, thus LTP inhibition is not caused by a change in the glutamatergic receptor-mediated currents in pyramidal neurons of the CA1 region.
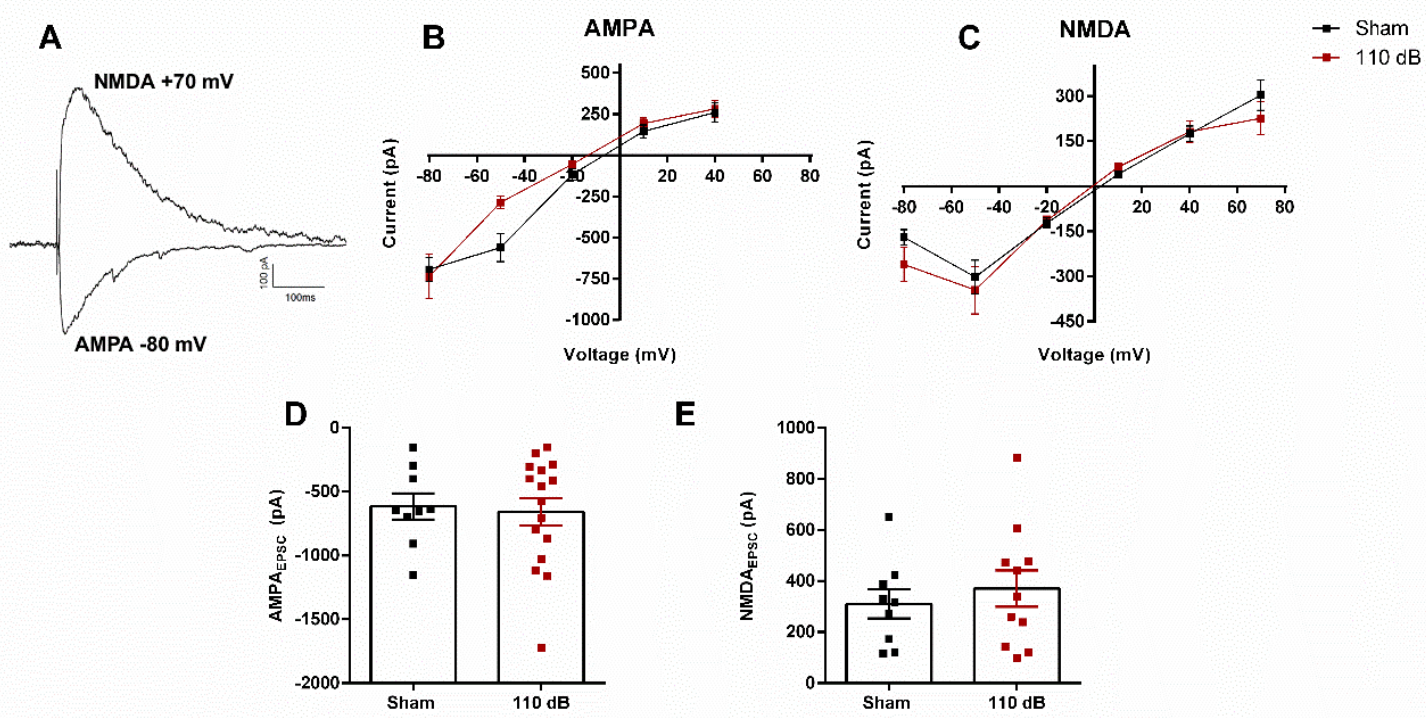

E

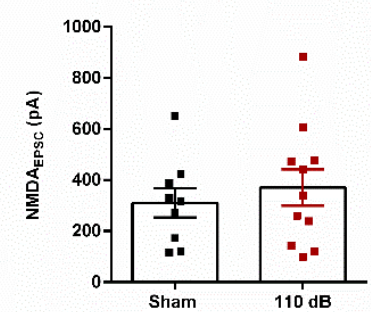

Figure 2. Glutamatergic neurotransmission evoked by AMPA/KA and NMDA receptors. (A) Representative traces of AMPA/KA currents (DNQX-sensitive) at $-80 \mathrm{mV}$ and NMDA currents (DNQX-resistant) at $+70 \mathrm{mV}$, respectively. AMPA/KA (B) and NMDA (C) peak currents at different voltages. Mean peak currents of AMPA/KA (D) at $-80 \mathrm{mV}$ and NMDA at $+70 \mathrm{mV}(\mathrm{E})$.

High-intensity sound exposure does not substantially affect GABAergic transmission in CA1 pyramidal neurons.

Previous results from our group showed that a long-term protocol of high-intensity sound stimulation which inhibits LTP in the Schaffer-CA1 synapse (2 episodes a day, for 10 days; Cunha et al., 2015) potentiates GABAergic transmission on pyramidal CA1 hippocampal neurons (Cunha et al., 2019) by increasing the amplitude of the GABAergic currents. We then tested if a single episode of high-intensity sound could potentiate the GABAergic neurotransmission in the CA1 pyramidal neurons.

However, different to what observed with the 10-days protocol we did not observe changes in the GABAergic transmission (Figure $3 \mathrm{~A}$ ) in the hippocampus of animals subjected to one minute of high-intensity sound. Moreover, we did not find differences in the amplitude of the mIPSCs after the application of TTX: (sham stimulated: $75.77 \pm 6.1 \mathrm{pA}, \mathrm{n}=8$; stimulated: $69.63 \pm 3.52 \mathrm{pA}, \mathrm{n}=8 ; \mathrm{P}=0.39$, Figure $3 \mathrm{~B}$ ). We found a smaller, but no significant reduction in the frequency of the mIPSCs in neurons from stimulated animals (sham: $2.05 \pm 0.48 \mathrm{~Hz}, \mathrm{n}=8$, stimulated: $1.15 \pm 0.16 \mathrm{~Hz}, \mathrm{n}=9, \mathrm{P}=0.08$, Figure $3 \mathrm{E}$ ). However, we found that mIPSCs from stimulated animals have faster decay times than the mIPSCs from control animals (fast decay time: sham: $2.9 \pm 0.12 \mathrm{~ms} ; \mathrm{n}=8$; stimulated: $2.4 \pm 0.18 \mathrm{~ms} ; \mathrm{n}=9$; $\mathrm{P}=0.04$, Figure $3 \mathrm{H}$; slow decay time: sham: $25.04 \pm 1.22 \mathrm{~ms}, \mathrm{n}=8$ and stimulated: $20.42 \pm 0.90 \mathrm{~ms}, \mathrm{n}=9 ; \mathrm{P}=0.003$, Figure $3 \mathrm{l}$ ). The proportion of the two components (fast/slow) was similar in both groups (sham: $53 \%$; stimulated: $48 \%$, Figure $3 \mathrm{~J}$ ). We conclude that, contrary to the observed after the long-term stimulation with high-intensity sound, the GABAergic inhibitory neurotransmission is not potentiated by a short stimulus with high-intensity sound, but a similar change in current kinetics was observed. 

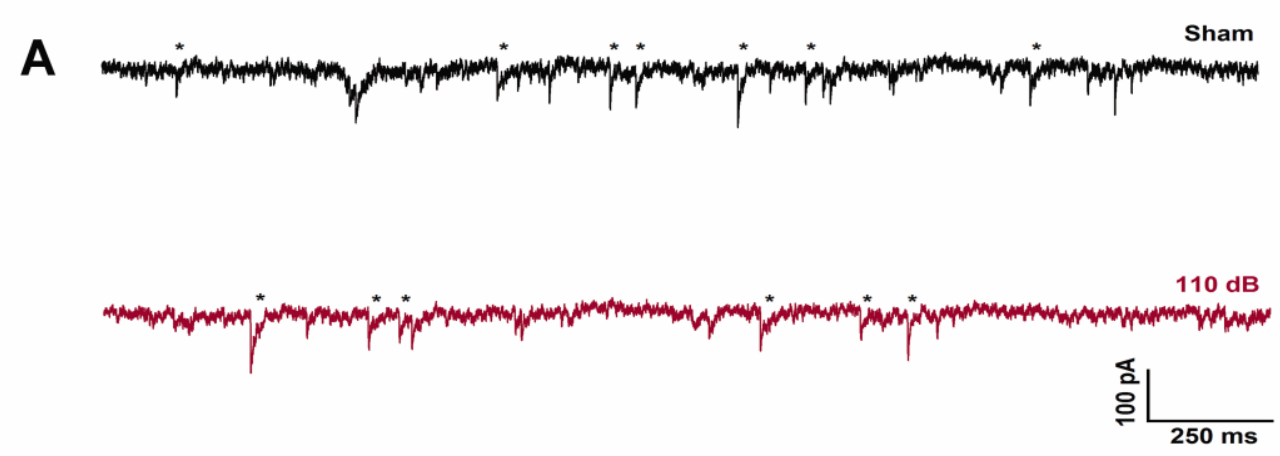
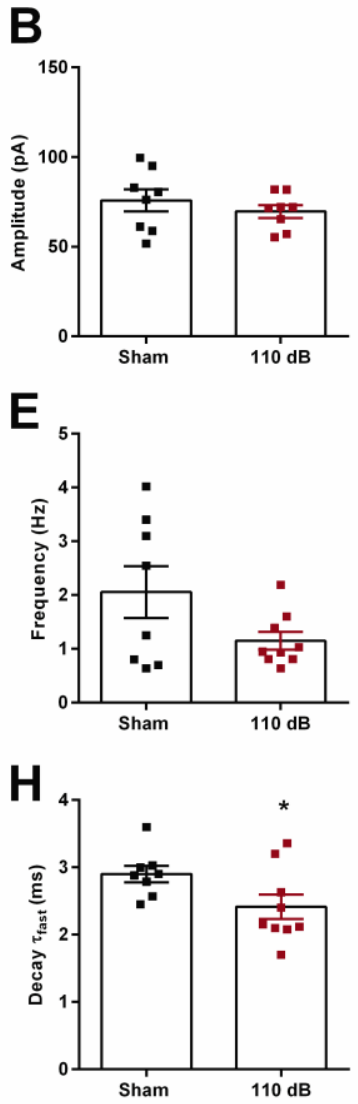

C
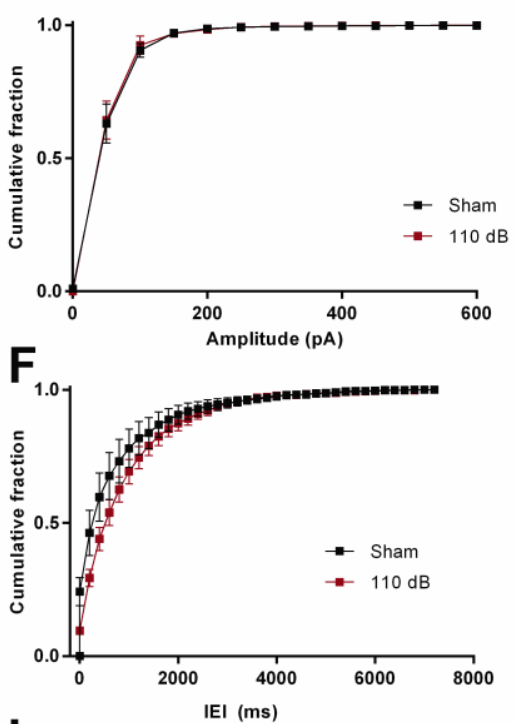

I

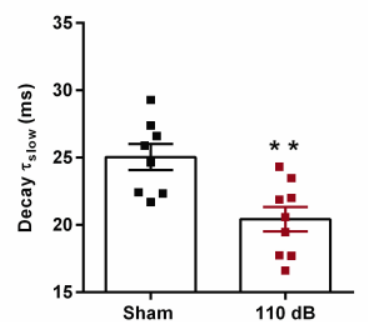

D
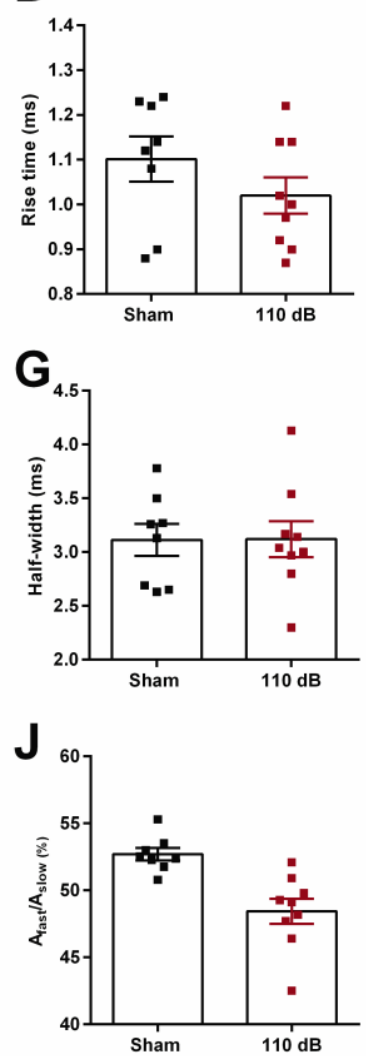

Figure 3. Miniature GABAergic currents. A) Representative traces of inhibitory currents from sham-stimulated rats (black trace) and rats submitted to a sound stimulus of $110 \mathrm{~dB}$ (red trace). B) Mean amplitude of events detected and C) cumulative fraction of amplitudes per group. D) Mean rise time of mIPSC. E) Mean frequency and F) Cumulative fraction of IEI of mIPSC. G) Mean half-widths. (H) Mean fast and (I) slow decay time constants. J) The ratio between $A_{\text {fast }}$ and $A_{\text {slow }}$ shown as a percentage. ${ }^{*} \mathrm{P}<0.05{ }^{*} \mathrm{P}<0.01$.

High-intensity sound exposure decreases BDNF levels in the dorsal hippocampus, and the inhibition of LTP is reverted by application of BDNF or its receptor agonist.

Given that we observed no changes in the glutamatergic and inhibitory currents, we tested if another factor could account for the reduction in hippocampal LTP in these animals. Because of the strong effect of BDNF in facilitating hippocampal LTP, we hypothesized that highintensity sound exposure could affect BDNF production or secretion in the hippocampus. Indeed, we found that BDNF levels in dorsal hippocampus are significantly decreased in 
stimulated (1732 \pm 76.87 pg.mg protein $\left.{ }^{-1} ; n=7\right)$ in comparison with sham rats ( $2344 \pm 89.06$ pg.mg protein ${ }^{-1}, n=8 ; P=0.0002$ ) (Figure 4). Thus, our results show that high-intensity sound decreases secretion or production of BDNF in the hippocampus.

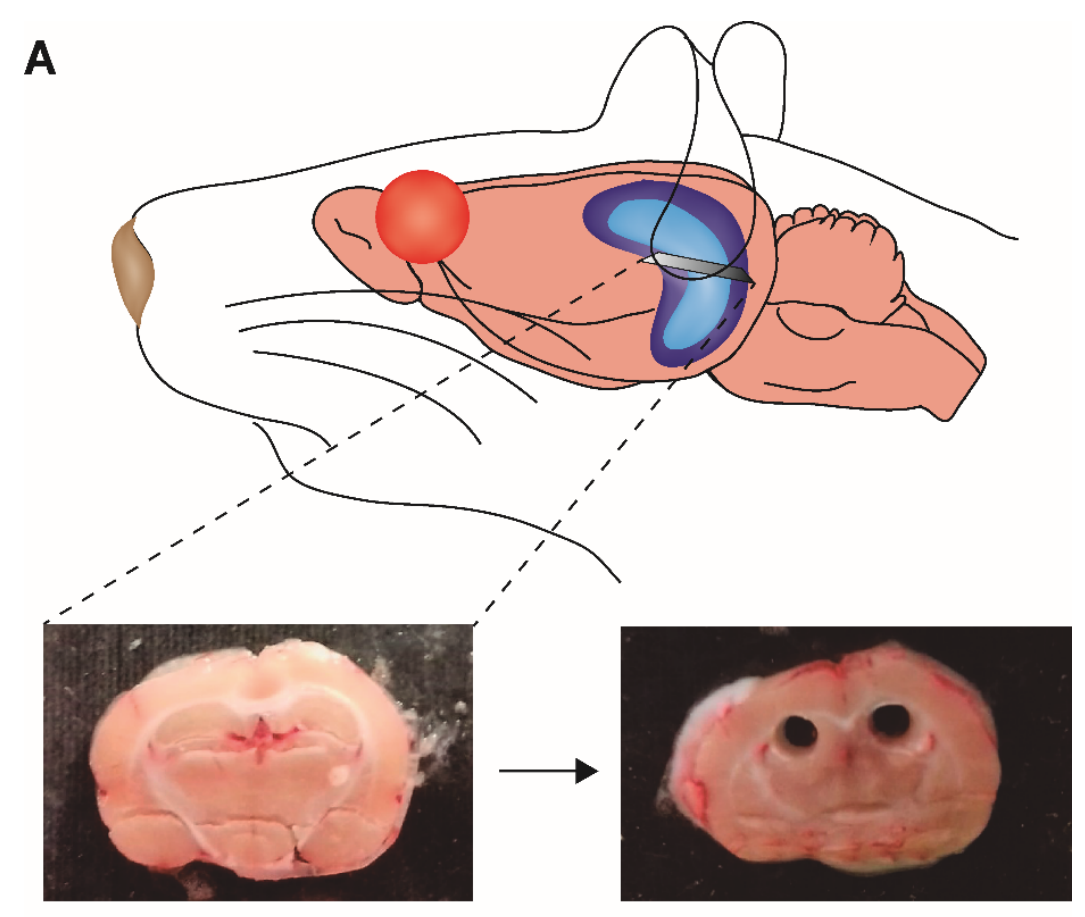

B

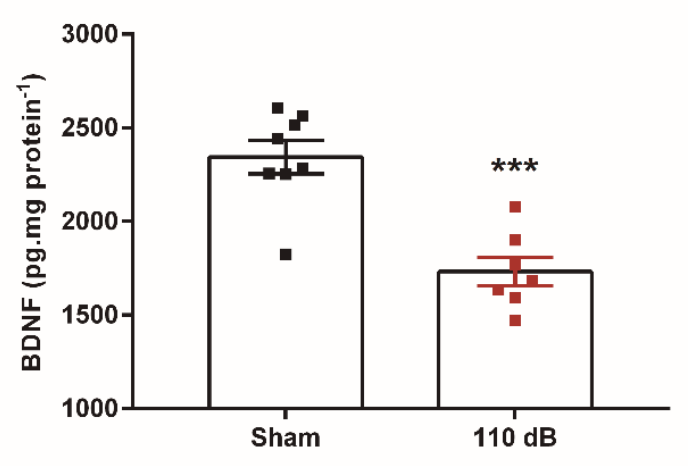

Figure 4. A) Schematic drawing of a transversal slice of hippocampus. B) Representative photo of bilateral dorsal hippocampus before and after the punch $(1200 \mu \mathrm{m})$. C) BDNF levels in the dorsal hippocampus from sham-stimulated and rats submitted to a sound stimulus of $110 \mathrm{~dB}$. $* * * \mathrm{P}<0.005$.

We then decided to test if perfusion of BDNF to the hippocampal slices of rats exposed to high-intensity sound could revert the inhibition of LTP, similar to observed in the hippocampi of animal submitted to chronic intermittent hypoxia (Xie et al., 2010). First we reproduced our original observation (de Deus et al., 2017) that exposure to $110 \mathrm{~B}$ sound for one minute inhibits the LTP in the Schaffer-CA1 synapse (Figure 5A,B,C; sham: $1.41 \pm 0.11 ; n=4$; sound exposed: $1.04 \pm 0.06 ; n=5 ; P=0.01)$. Perfusion of BDNF $(25 \mathrm{ng} / \mathrm{ml})$ previously to the induction of LTP in the Schaffer-CA1 synapse in slices from sham animals did not change the magnitude of LTP (1.43 $\pm 0.07 ; n=7$. $P=0.90$. Figure $5 \mathrm{D}, \mathrm{E}$ ) showing that supplementation of BDNF to the hippocampus of animals not subjected to high-intensity sound exposure does not. However, when we 
perfused the slices of rats submitted to one minute of $110 \mathrm{~dB}$ sound with BDNF, we observed a recovery of LTP $(1.55 \pm 0.12 ; n=6 ; P=0.01)$ when compared to sound exposed group, to the same level of the sham group ( $P=0.47$, Figure $5 F)$. Additionally, we tested if the Trk-B agonist LM22A4 could restore hippocampal LTP in slices of sound-exposed rats. Indeed, perfusion of LM22A4 $(5 \mu \mathrm{M})$ rescued hippocampal LTP from slices from sound exposed rats $(1.62 \pm 0.17 ; n=$ 5; $P=0.02$ compared with the sound exposed group) to the same level to the LTP observed in slices from sham rats; $p=0.38$; Figure $5 \mathrm{G}, \mathrm{H}$ ). These results indicate that Trk-B receptor activation by BDNF reverts the LTP inhibition caused by exposure to high-intensity sound. Together with the reduced levels of BDNF in the hippocampus of these animals, these results suggest that exposition to on minute of high-intensity sound decreases BDNF levels in the hippocampal CA1 region resulting in inhibition of LTP induction.

A
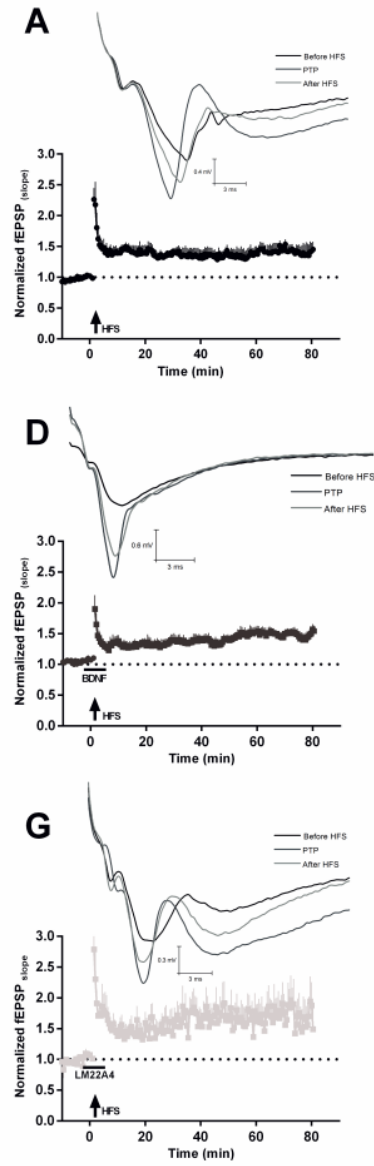

B

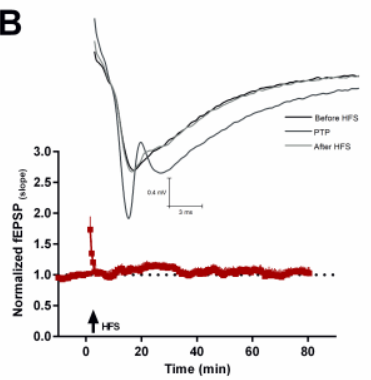

E

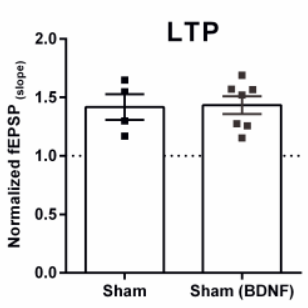

C
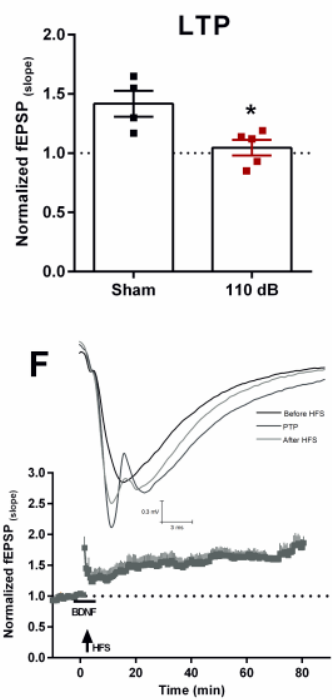

H

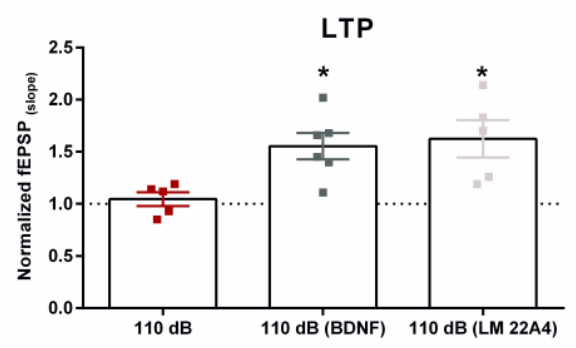

Figure 5. LTP from the Schaffer-CA1 synapse of sham- stimulated rats $(A)$ and animals submitted $110 \mathrm{~dB}$ sound exposure (B). Normalized fEPSP slopes before and after HFS (arrow) from the Schaffer-CA1 synapse of slices of sham animals and stimulated rats. C) Bar graphs showing the summary of LTP for sham-stimulated and stimulated rats. D) Normalized fEPSP slopes before and after HFS (arrow) from the Schaffer-CA1 synapse of slices treated with BDNF (bar-10 minutes) from sham-stimulated rats. E) Bar graph showing the summary of LTP for shamstimulated and stimulated rats. F) Normalized fEPSP slopes before and after HFS (arrow) from the Schaffer-CA1 synapses of slices treated with BDNF (bar-10 minutes) from stimulated rats. (G) Normalized fEPSP slopes before and after HFS (arrow) from the Schaffer-CA1 synapse of slices treated with LM22A4 (bar-10 minutes) from stimulated rats. Bar graph showing the summary of LTP from control slices and slices treated with BDNF and LM22A4 from stimulated rats. Representative recordings are shown above each graph. ${ }^{*} \mathrm{P}<0.05$. 


\section{Discussion}

Several lines of evidence show that auditory stimulation has effects beyond the auditory processing areas (Lercher et al., 2003; Stansfeld et al., 2005; Uran et al., 2010, 2012; Kraus and Canlon, 2012; Basner et al., 2014). Especially, intense auditory stimulation has been shown by us to interfere with long-term potentiation in the hippocampal Schaffer-CA1 synapse (Cunha et al., 2015; de Deus et al., 2017). Our first observation was that a 10-day long protocol of two daily episodes of $120 \mathrm{~dB}$ sound exposure for 1 minute each was able to inhibit the LTP in the hippocampal Schaffer-CA1 synapse of Wistar rats (Cunha et al., 2015). We chose this protocol because we were initially interested in testing if animals susceptible to audiogenic seizures from the WAR (Wistar Audiogenic Rats) strain (Doretto et al., 2003) had abnormal LTP after the protocol of audiogenic kindling (Marescaux et al., 1987; Naritoku et al., 1992; Garcia-Cairasco et al., 1996) which consisted of the exposure of the animals with the sound protocol above mentioned. To our surprise, we found that the rats from WAR strain, which consistently developed limbic seizures during the protocol of audiogenic kindling, did not have changes in LTP (Cunha et al., 2015). On the other hand, LTP in the Schaffer-CA1 synapse in the hippocampus of control Wistar rats, which did not develop audiogenic limbic seizures, was impaired (Cunha et al., 2015). We later found that a single one-minute episode of $110 \mathrm{~dB}$ sound was able to inhibit LTP in the Schaffer-CA1 synapse (de Deus et al., 2017), showing that exposure to high-intensity sound even for a brief period can impact hippocampal LTP. Interestingly, despite this strong effect in inhibiting LTP we did not find any deficit in spatial navigation and memory in the animals subjected to our protocols of high intensity sound exposure (Cunha et al., 2015; de Deus et al., 2017), showing that despite inhibiting hippocampal LTP, exposure to high-intensity sound does not affect basic spatial learning and memory processes.

In order to investigate the possible mechanisms of this effect on the hippocampi of Wistar rats, we performed experiments studying the synaptic transmission on the CA1 pyramidal neurons and their intrinsic electrophysiological properties (Cunha et al., 2018, 2019). The mechanisms of the inhibition of LTP by the prolonged exposure to high-intensity sound might be related, at least partially, to an increase in membrane resistance and decrease in action potential threshold, by a reduction in the expression of $\mathrm{I}_{\mathrm{h}}$ in CA1 pyramidal neurons (Cunha et al., 2018) and a potentiation of GABAergic transmission (Cunha et al., 2019). However, in the animals subjected to one minute of high-intensity sound, we did not find differences in the intrinsic properties of CA1 pyramidal neurons (Cunha et al., 2018) showing that the impact of high-intensity sound in the hippocampus is dependent on the length of sound exposure.

In this work, we continued the investigation of the mechanisms of LTP inhibition after one-minute exposure to a $110 \mathrm{~dB}$ sound. We found like observed after prolonged exposure to one-minute episodes of $110 \mathrm{~dB}$ sound (Cunha et al., 2019) that the glutamatergic transmission both via AMPA/kainite and NMDA receptors are not affected by a one minute $110 \mathrm{~dB}$ sound exposure. However, no potentiated GABAergic transmission was observed after a single episode of $110 \mathrm{~dB}$ sound, differently to the potentiated GABAergic transmission after ten days of stimulation (Cunha et al., 2019). This suggests that the potentiated GABAergic transmission is a late response to high-intensity sound stimulation and probably compensates the late increased excitability of CA1 pyramidal neurons observed after the prolonged exposure to high-intensity sound (Cunha et al., 2018). Thus, this indicates that the effects of high-intensity sound are distinct in both models of high-intensity sound exposure.

The hippocampus is connected to the auditory system indirectly from the frontomedial cortex, insula, and amygdala (Kraus and Canlon, 2012), by a pathway from the cochlear nucleus to the entorhinal cortex involving the pontine reticula nucleus, pontine central gray and medial septum, with a higher threshold than the canonical pathway via inferior colliculus (Zhang et al., 2018) and by a direct connection between the auditory cortex and the CA1 region (Zhao et al., 
2018). This pathway is implicated in the formation of long-term auditory and auditory-spatial memories (Squire et al., 2001; Tamura et al., 1990; Arononv et al., 2017) and fear conditioning (Zhang et al., 2018). Accordingly, we showed that c-FOS expression is increased in the pyramidal cell layer of both CA1 and CA3 regions. C-FOS expression is induced by strong increases in intracellular calcium trough NMDA receptors and voltage-dependent calcium channels, which activates MAPK pathway leading to the phosphorylation of the transcription factors CREB and Elk-1, that bind to the c-fos promoter (Chung, 2015; Hudson, 2018). The expressed FOS protein dimerizes with the protein JUN to form the AP-1 complex that regulates the expression of several genes that could be involved in the inhibitory effect of high-intensity sound on LTP. Because c-fos expression needs strong calcium influx induced by synaptic activity, we could hypothesize that high-intensity sound stimulation induces a hyperexcitation on hippocampal neurons during the exposure to sound. We found increased c-fos expression in the pyramidal layer, were the glutamatergic pyramidal neurons are located, however we cannot discard the activation of GABAergic interneurons located in the pyramidal layer or adjacent to it. Interestingly, in vivo hippocampal recordings showed that sound exposure evokes inhibitory currents on pyramidal neurons, accordingly to the activation of hippocampal inhibitory interneurons (Wang et al., 2017).

Based on the critical role of BDNF over hippocampal LTP (Minichielo, 2009; Edelmann et al., 2014; Lin et al., 2018), we tested the hypothesis that a lack of BDNF could be related to the LTP impairment after high-intensity sound exposure. We found that the expression of the mature form of BDNF was reduced in the hippocampus of rats exposed to high-intensity sound. This could represent a depletion of the cellular BDNF and/or increasing degradation of existing BDNF.We then demonstrated that exogenous application of BDNF was able to rescue the LTP from the Schaffer-CA1 synapses from rats exposed to high-intensity sound. Because BDNF did not increase the magnitude of LTP from the Schaffer-CA1 synapses from sham rats, the increase in the magnitude of LTP in sound exposed animals was not due to a facilitation of LTP by exogenous BDNF, but we believe it represented a replenishment of reduced BDNF levels in the hippocampus of these animals. Additionally, the agonist of the Trk-B receptor LM22A4 also rescued the LTP from sound stimulated animals, confirming that the hippocampal BDNF/ Trk-B pathway is affected by high-intensity sound exposure. However, the efficacy of LM22A4 and other small molecule agonists of Trk-B receptors has been recently questioned (Boltaev et al., 2017). Thus, we conclude that a decrease in BNDF levels in the hippocampus of rats submitted to one minute of high-intensity sound, impairs the expression of LTP in the Schaffer-CA1 synapse. Interestingly, Matt et al., (2018) found that prolonged exposure to traumatic noise (120 $\mathrm{dB}, 6$ hours per day for 21 days) in anesthetized rats did not alter LTP and BDNF expression. This suggests that the anesthesia or the prolonged sound exposure protocol might change the hippocampal response to high-intensity sound. We do not know, however, if the same decrease in BDNF levels occurs in the hippocampus of animals subjected to the 10-days long sound exposure protocol.

Our results add to other reports showing the influence of the acoustic environment on hippocampal function. Both moderate and intense sound exposure affect hippocampal function in several ways. For instance prolonged ( 2 hours/ day for 3-6 weeks) moderate ( $80 \mathrm{~dB}$ ) sound exposure, impairs spatial memory in mice and increases oxidative damage and hippocampal tau phosphorylation (Cheng et al., 2011, 2016). On the other hand, daily exposure to music at moderated intensity levels ( $60 \mathrm{~dB}, 6$ hours per day for 21 days) enhanced learning performance and increased BDNF expression in the hippocampus (Angelucci et al., 2007). Similarly, exposure to an $80 \mathrm{~dB}$ sound at $10 \mathrm{kHz}$ for 40 minutes in anesthetized rats, increased BDNF expression, hippocampal LTP magnitude and improved the performance in the Morris water maze (Matt et al., 2018). It appears that the effect of moderate noise is more related to the type of noise, and it seems to be contradictory depending on the reports. Exposure to intense noise, on the other 
hand, is often associated with deficits in hippocampal function. For instance, acute traumatic noise (106-115 dB, 30-60 minutes) alters place cell activity in the hippocampus and increases arc expression, an immediate early gene related to synaptic plasticity, in the hippocampi of rats. Prenatal exposure to loud sounds has a deleterious effect on the hippocampal LTP hippocampaldependent learning and memory in rats (Barzegar et al., 2015). Sound deprivation has also been shown to impact hippocampal function. Zhao et al., (2018) showed that temporary conductive hearing loss in young rats reduced LTP, NMDA currents, dendritic spine density and resulted in impaired performance in the Morris water maze 30 days later when the hearing was restored.

Thus, the results presented here and in the other reports from our group, in combination with the above-mentioned evidences, show that the hippocampus is strongly influenced by the auditory environment and that the BDNF is an important mediator of these effects. The knowledge about the influence of noise exposure on non-auditory areas like the hippocampus is extremely relevant because exposure to loud noises is an increasingly common occurrence in our daily life, and several cognitive and emotional deficits are associated to prolonged and acute noise exposure in humans (Lercher et al., 2003; Stansfeld et al., 2005; Basner et al., 2014; Hofner et al., 2018; Swanson et al., 2018).

Acknowledgments: Work supported by São Paulo State Research Foundation (FAPESP) grants 2015/22327-7, 2016/01607-4 and 2016/17681-9. We thank the technical assistance of Mr. J. Fernando Aguiar and Mr. Rubens Fernando de Melo.

\section{References}

Aicardi G, Argilli E, Cappello S, Santi S, Riccio M, Thoenen H, Canossa M (2004) Induction of long-term potentiation and depression is reflected by corresponding changes in secretion of endogenous brain-derived neurotrophic factor. Proceedings of the National Academy of Sciences of the United States of America 101: 15788-15792.

Angelucci F, Fiore M, Ricci E, Padua L, Sabino A, Tonali PA (2007) Investigating the neurobiology of music: brain-derived neurotrophic factor modulation in the hippocampus of young adult mice. Behav Pharmacol 18: 491-6.

Aronov D, Nevers R, Tank DW (2017) Mapping of a non-spatial dimension by the hippocampal-entorhinal circuit. Nature 543:719-722.

Barzegar M, Sajjadi FS, Talaei SA, Hamidi G, Salami M (2015) Prenatal exposure to noise stress: anxiety, impaired spatial memory, and deteriorated hippocampal plasticity in postnatal life. Hippocampus 25: 187-96.

Basner M, Babisch W, Davis A, Brink M, Clark C, Janssen S, Stansfeld S (2014) Auditory and non-auditory effects of noise on health. Lancet 383: 1325-32.

Bliss TVP, Lomo T (1973) Long-lasting potentiation of synaptic transmission in the dentate area of the anaesthetized rabbit following stimulation of the perforant path. J Physiol (Lond) 232:331-356.

Bliss TVP, Collingridge GL (1993) A synaptic model of memory - long-term potentiation in the hippocampus. Nature 361: 31-39. 
Boltaev U, Meyer Y, Tolibzoda F, Jacques T, Gassaway M, Xu Q, Wagner F, Zhang YL, Palmer M, Holson E, Sames D (2017) Multiplex quantitative assays indicate a need for reevaluating reported small-molecule TrkB agonists. Sci Signal. 10(493).

Burow A, Day H, Campeau S (2005) A detailed characterization of loud noise stress: Intensity analysis of hypothalamo-pituitary-adrenocortical axis and brain activation. Brain Research 1062:63-73.

Cheng L, Wang SH, Chen QC, Liao XM (2011) Moderate noise induced cognition impairment of mice and its underlying mechanisms. Physiol Behav 104:981-988.

Cheng L, Wang SH, Huang Y, Liao XM (2016) The hippocampus may be more susceptible to environmental noise than the auditory cortex. Hear Res 333:93-97.

Chung L (2015) A brief introduction to the transduction of neural activity into Fos signal. Development \& Reproduction 19: 61-67.

Collingridge GL, Bliss TVP (1987) NMDA receptors- their role in long-term potentiation. Trends Neurosci 7:288-293.

Cunha AO, de Oliveira JA, Almeida SS, Garcia-Cairasco N, Leão RM (2015) Inhibition of long-term potentiation in the Schaffer-CA1 pathway by repetitive high-intensity sound stimulation. Neuroscience 310:114-27.

Cunha AOS, Ceballos CC, de Deus JL, Leão RM (2018) Long-term high-intensity sound stimulation inhibits h current (Ih) in CA1 pyramidal neurons. Eur J Neurosci 11:1401-1413.

Cunha AOS, de Deus JL, Ceballos CC, Leão RM (2019) Increased hippocampal GABAergic inhibition after long-term high-intensity sound exposure. PLoS One 5:e0210451.

de Deus JL, Cunha AOS, Terzian AL, Resstel LB, Elias LLK, Antunes-Rodrigues J, Almeida SS, Leão RM (2017) A single episode of high intensity sound inhibits long-term potentiation in the hippocampus of rats. Sci Rep 1:14094.

Doretto MC, Fonseca CG, Lôbo RB, Terra VC, Oliveira JA, Garcia-Cairasco N (2003) Quantitative study of the response to genetic selection of the Wistar audiogenic rat strain (WAR). Behav Genet 33: 33-42.

Dragunow M, Faull R (1989) The use of c-fos as a metabolic marker in neuronal pathway tracing. J Neurosci Methods 3:261-5.

Edelmann E, Cepeda-Prado E, Franck M, Lichtenecker P, Brigadski T, Leßmann V (2015) Theta Burst Firing Recruits BDNF Release and Signaling in Postsynaptic CA1 Neurons in SpikeTiming-Dependent LTP. Neuron 4:1041-1054.

Eggermont JJ (2017) Effects of long-term non-traumatic noise exposure on the adult central auditory system. Hearing problems without hearing loss. Hear Res 352:12-22.

Garcia-Cairasco N, Wakamatsu H, Oliveira JAC, Gomes ELT, Del Bel EA, Mello LEAM (1996) Neuroethological and morphological (Neo-Timm staining) correlates of limbic recruitment during the development of audiogenic kindling in seizure susceptible Wistar rats. Epilepsy Res 26, 177-192.

Goble TJ, Møller AR, Thompson LT (2009) Acute high-intensity sound exposure alters responses of place cells in hippocampus. Hear Res 253:52-59. 
Helfferich F, Palkovits, M (2003) Acute audiogenic stress-induced activation of CRH neurons in the hypothalamic paraventricular nucleus and catecholaminergic neurons in the medulla oblongata. Brain Res 975:1-9.

Hoffer ME, Levin BE, Snapp H, Buskirk J, Balaban C (2018) Acute findings in an acquired neurosensory dysfunction. Laryngoscope Investig Otolaryngol. 4:124-131.

Hudson AE (2018) Genetic Reporters of Neuronal Activity: c-Fos and G-CaMP6. Methods Enzymol 603:197-220.

Jeffery KJ (2007) Integration of the sensory inputs to place cells: what, where, why, and how? Hippocampus 17: 775-785.

Korte M, Carroll P, Wolf E, Brem G, Thoenen H, Bonhoeffer T (1995) Hippocampal longterm potentiation is impaired in mice lacking brain-derived neurotrophic factor. Proc. Natl Acad 92: 8856-8860.

Kraus KS, Canlon B (2012) Neuronal connectivity and interactions between the auditory and limbic systems. Effects of noise and tinnitus. Hear Res 288:34-46.

Le TN, Straatman LV, Lea J, Westerberg B (2017) Current insights in noise-induced hearing loss: a literature review of the underlying mechanism, pathophysiology, asymmetry, and management options. J Otolaryngol Head Neck Surg 1:41.

Lercher P, Evans GW, Meis M (2003) Ambient noise and cognitive processes among primary schoolchildren. Environment and Behavior 35: 725-735.

Lin PY, Kavalali ET, Monteggia LM (2018) Genetic Dissection of Presynaptic and Postsynaptic BDNF-TrkB Signaling in Synaptic Efficacy of CA3-CA1 Synapses. Cell Rep 6:15501561.

Malenka RC, Bear MF (2004) LTP and LTD: an embarrassment of riches. Neuron 1:5-21.

Manikandan S, Padmab MK, Srikumar R, Parthasarathy NJ, Muthuvel A, Sheela Devi R (2006) Effects of chronic noise stress on spatial memory of rats in relation to neuronal dendritic alteration and free radical-imbalance in hippocampus and medial prefrontal cortex. Neurosci. Lett 399: 17-22.

Manohar S, Spoth J, Radziwon K, Auerbach BD, Salvi R (2017) Noise-induced hearing loss induces loudness intolerance in a rat Active Sound Avoidance Paradigm (ASAP). Hear Res 353:197-203.

Marescaux C, Vergnes M, Kiesmann M, Depaulis A, Micheletti G, and Warter JM (1987) Kindling of Audiogenic Seizures in Wistar Rats: An EEG Study. Exp Neurol 97:160- 168.

Matt L, Eckert P, Panford-Walsh R, Geisler HS, Bausch AE, Manthey M, Müller NIC, Harasztosi C, Rohbock K, Ruth $P$, Friauf E, Ott T, Zimmermann U, Rüttiger L, Schimmang $T$, Knipper M, Singer W (2018) Visualizing BDNF transcript usage during sound-induced memory linked plasticity. Front Mol Neurosci. 11: 260.

Minichielo L (2009) TrkB signaling pathways in LTP and learning. Nat Rev Neurosci 12:850-60.

Naritoku DK, Mecozzi LB, Aiello MT, Faingold CL (1992) Repetition of audiogenic seizures in genetically epilepsy-prone rats induces cortical epileptiform activity and additional seizure behaviors. Exp Neurol 115:317-324.

Nicoll, RA (2017) A Brief History of Long-Term Potentiation. Neuron 2:281-290. 
Patterson SL, Abel T, Deuel TA, Martin KC, Rose JC, Kandel ER (1996) Recombinant BDNF rescues deficits in basal synaptic transmission and hippocampal LTP in BDNF knockout mice. Neuron 16:1137-1145.

Ravassard P, Kees A, Willers B, Ho D, Aharoni D, Cushman J, Aghajan ZM, Mehta MR (2013) Multisensory control of hippocampal spatiotemporal selectivity. Science 340: 1342-1346.

Romcy-Pereira RN, Garcia-Cairasco N (2003) Hippocampal cell proliferation and epileptogenesis after audiogenic kindling are not accompanied by mossy fiber sprouting or Fluoro-Jade staining. Neuroscience 119:533-46.

Save E, Nerad L, Poucet B (2000) Contribution of multiple sensory information to place field stability in hippocampal place cells. Hippocampus 10: 64-76.

Squire LR, Schmolck H, Stark SM (2001) Impaired auditory recognition memory in amnesic patients with medial temporal lobe lesions. Learn Mem 8:252-6.

Stansfeld SA, Berglund B, Clark C, Lopez-Barrio I, Fischer P, Ohrström E, Haines MM, Head J, Hygge S, van Kamp I, Berry BF; RANCH study team (2005) Aircraft and road traffic noise and children's cognition and health: a cross-national study. Lancet 365: 1942-9.

Swanson RL 2nd, Hampton S, Green-McKenzie J, Diaz-Arrastia R, Grady MS, Verma R, Biester R, Duda D, Wolf RL, Smith DH (2018) Neurological manifestations among US government personnel reporting directional audible and sensory phenomena in Havana, Cuba. JAMA. 319:1125-1133.

Tamura R, Ono T, Fukuda M, Nakamura K (1990) Recognition of egocentric and allocentric visual and auditory space by neurons in the hippocampus of monkeys. Neurosci. Lett 109:293-298.

Tang YP, Shimizu E, Dube GR, Rampon C, Kerchner GA, Zhuo M, Liu G, Tsien JZ (1999) Genetic enhancement of learning and memory in mice. Nature 401: 63-69.

Tsien JZ, Huerta PT, Tonegawa S (1996) The essential role of hippocampal CA1 NMDA receptor-dependent synaptic plasticity in spatial memory. Cell 87: 1327-1338.

Uran SL, Caceres LG, Guelman LR (2010) Effects of loud noise on hippocampal and cerebellar-related behaviors. Role of oxidative state. Brain Res 1361:102-14.

Uran SL, Aon-Bertolino ML, Caceres LG, Capani F, Guelman LR (2012) Rat hippocampal alterations could underlie behavioral abnormalities induced by exposure to moderate noise levels. Brain Res 1471:1-12.

Wang N, Gan X, Liu Y, Xiao Z (2017) Balanced Noise-Evoked Excitation and Inhibition in Awake Mice CA3. Front Physiol 8:931.

Xie H, Leung KL, Chen L, Chan YS, Ng PC, Fok TF, Wing YK, Ke Y, Li AM, Yung WH (2010) Brain-derived neurotrophic factor rescues and prevents chronic intermittent hypoxia-induced impairment of hippocampal long-term synaptic plasticity. Neurobiol Dis 40:155-62.

Zhang GW, Sun WJ, Zingg B, Shen L, He J, Xiong Y, Tao HW, Zhang LI. (2018) A Noncanonical Reticular-Limbic Central Auditory Pathway via Medial Septum Contributes to Fear Conditioning. Neuron. 97:406-417

Zhao H, Wang L, Chen L, Zhang J, Sun W, Salvi RJ, Huang YN, Wang M, Chen L (2018) Temporary conductive hearing loss in early life impairs spatial memory of rats in adulthood. Brain Behav 7:e01004. 
bioRxiv preprint doi: https://doi.org/10.1101/850214; this version posted November 21, 2019. The copyright holder for this preprint (which was not certified by peer review) is the author/funder, who has granted bioRxiv a license to display the preprint in perpetuity. It is made available under aCC-BY-NC-ND 4.0 International license. 